\title{
Resiliencia socio-ecológica: una perspectiva teórico- metodológica para el turismo comunitario
}

\author{
Fernando David Chontasi Morales ${ }^{1,2}$, Jessica Dayana Noguera Benalcázar ${ }^{3}$, \\ Daniela Pilar Ortega Váscone $z^{4}$, Mery Tatiana Chicaiza Guaman', \\ Luis Arturo Naula Morillo ${ }^{6}$, Diana Carolina Duarte Victorero ${ }^{7}$
}

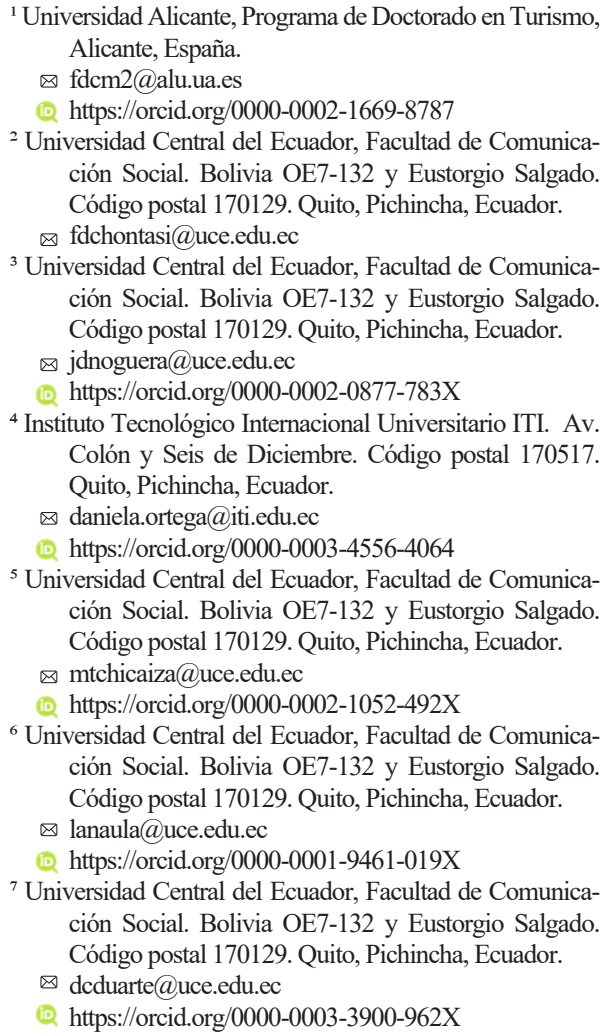

${ }^{2}$ Universidad Central del Ecuador, Facultad de Comunicación Social. Bolivia OE7-132 y Eustorgio Salgado. Código postal 170129. Quito, Pichincha, Ecuador. 凶fdchontasi@uce.edu.ec

${ }^{3}$ Universidad Central del Ecuador, Facultad de Comunicación Social. Bolivia OE7-132 y Eustorgio Salgado. Código postal 170129. Quito, Pichincha, Ecuador. Colón y Seis de Diciembre. Código postal 170517. Quito, Pichincha, Ecuador.

${ }^{5}$ Universidad Central del Ecuador, Facultad de Comunicación Social. Bolivia OE7-132 y Eustorgio Salgado. Código postal 170129. Quito, Pichincha, Ecuador.

${ }^{6}$ Universidad Central del Ecuador, Facultad de Comunicación Social. Bolivia OE7-132 y Eustorgio Salgado. Código postal 170129. Quito, Pichincha, Ecuador.

凶1anaula@uce.edu.ec

6 https://orcid.org/0000-0001-9461-019X

${ }^{7}$ Universidad Central del Ecuador, Facultad de Comunicación Social. Bolivia OE7-132 y Eustorgio Salgado. Código postal 170129. Quito, Pichincha, Ecuador.

凶 dcduarte@uce.edu.ec

(D. https://orcid.org/0000-0003-3900-962X

*Autor de correspondencia: fdchontasi@uce.edu.ec

\section{SIEMBRA}

https://revistadigital.uce.edu.ec/index.php/SIEMBRA ISSN-e: $2477-8850$

\section{ISSN: $1390-8928$}

Periodicidad: semestral

vol. 8, núm. 2, 2021

siembra.fag@uce.edu.ec

DOI: https://doi.org/10.29166/siembra.v8i2.2967

Esta obra está bajo una licencia internacional Creative Commons Atribución-NoComercial

\section{Resumen}

La resiliencia es una capacidad de las localidades turísticas por medio de la cual lidian con el cambio y las perturbaciones que ponen en peligro los recursos de vida. Los objetivos de este artículo son analizar los aportes teórico-metodológicos de la resiliencia en el turismo y diseñar un marco analítico, integral y flexible con el propósito de estimar la resiliencia de forma cualitativa en comunidades locales turísticas. Además, intenta servir de guía a investigadores que necesitan dar precisión a sus experiencias empíricas. Metodológicamente, se recurre a la revisión de la literatura especializada, con ello se examina el aporte recíproco de los sistemas socio-ecológicos y la resiliencia en el turismo. Enseguida se utiliza el método de análisis-síntesis, con la intención de identificar la importancia y el alcance de los elementos de un marco teórico-metodológico. Luego, se unifican criterios semejantes, a fin de conectar relaciones y formar un todo como aporte al conocimiento científico. De acuerdo con los resultados, la capacidad de análisis de los marcos teórico-metodológicos se sustenta en las características de los sistemas socio-ecológicos, el uso de factores de resiliencia y el empleo de medidas operacionales de resiliencia.

Palabras clave: resiliencia, turismo de base local, marco referencial, comunidad, sistema socio-ecológico.

\begin{abstract}
Resilience is a capacity of tourism localities to cope with change and disturbances that threaten living resources. The objectives of this article are to analyze the theoretical and methodological contributions of resilience in tourism and to design a comprehensive and flexible analytical framework for the purpose of qualitatively estimating resilience in local tourism communities. In addition, it is intended to serve as a guide for novice researchers who need to give precision to their empirical experiences. Methodologically, a review of the specialized literature is used to review the reciprocal contribution of social-ecological systems and resilience in tourism. Next, the method of analysis-synthesis is used, with the intention of identifying the importance and scope of the elements of a theoretical-methodological framework. Then, similar criteria are unified, in order to connect relationships and form a whole with a contribution
\end{abstract}


to scientific knowledge. According to the results, the analytical capacity of the theoretical-methodological frameworks is based on the characteristics of social-ecological systems, the use of resilience surrogates, and the use of operational measures of resilience.

Keywords: resilience, community-based tourism, framework, community, social-ecological system.

\section{Introducción}

El enfoque de sistemas lineales defiende la función determinista y que los postulados teóricos y conceptuales se centran en condiciones de equilibrio, balance o conservación. Este paradigma en el pensamiento ambiental clásico sostiene que la naturaleza no cambia y se regula internamente por ciclos que retornan a una sola posición de equilibrio, donde los humanos son externos a los sistemas ecológicos (Pickett et al., 2004). Sin embargo, surge una nueva racionalidad ambiental, que pondera las interrelaciones e interacciones entre los sistemas ambientales y sociales como un todo (Scoones, 1999). El énfasis no está en alcanzar el equilibrio, sino comprender que el cambio activa las capacidades de los elementos del sistema para permanecer en el tiempo.

En el año 1973, surge el estudio seminal de la resiliencia como una alternativa al pensamiento de la estabilidad (Holling, 1973). Se trata de una perspectiva sistémica, holística y complementaria de las dimensiones social y ecológica. De esta forma, se vislumbró una nueva dinámica de sistema: la socio-ecológica (SSE). Ésta se constituye como una forma diferente de ver la realidad inmediata y objetiva de una localidad específica, porque se nutre de los aportes multidisciplinarios, la memoria, la creatividad y la diversidad de sus funciones, para convivir y afrontar momentos de incertidumbre y perturbación. La acción compleja y recíproca de lo socio-ecológico distingue dinámicas y configuraciones novedosas, con ello, observa el comportamiento de las relaciones entre sus factores internos y las influencias externas (Gunderson y Holling, 2002). Esta interacción heterogénea concede al ser humano el rol de generar conocimiento en la línea de reconfigurar relaciones justas e imparciales, con incidencia en los procesos de renovación, adaptación y resiliencia.

La teoría de la resiliencia demuestra su utilidad porque tiene mucho poder de explicación y comprensión de los impactos repentinos dentro del sistema (Becken, 2013). Los sistemas socio-ecológicos identifican la resiliencia como una capacidad para absorber perturbaciones y reorganizarse mientras experimenta cambios (Walker et al., 2004). Su intención es comprender el cambio e identificar aquellos elementos endógenos proactivos que ayudan a gestionar los recursos y una gobernabilidad adaptativa. Estos factores determinan su posibilidad de aplicación como una herramienta metodológica de análisis del comportamiento de un sistema dinámico, como el turismo comunitario (Ruiz-Ballesteros, 2011).

Los territorios locales, así como el turismo comunitario, son ejemplos inmejorables de un sistema socio-ecológico. Conocer hasta qué punto las relaciones dentro un sistema socio-ecológico local aumentan o disminuyen la resiliencia es uno de los retos que los científicos pretenden demostrar a través de estudios longitudinales. Las contribuciones teóricas en este campo son emergentes y significativas (Ruiz-Ballesteros, 2011), pero se necesitan marcos metodológicos capaces de traducir el conocimiento y ayudar a estimar la resiliencia socio-ecológica. Por lo tanto, los objetivos de este artículo son analizar los aportes teórico-metodológicos de la resiliencia en el campo del turismo e intentar deconstruir una herramienta metodológica integral, flexible, adecuada a las necesidades de los territorios locales. Metodológicamente, se recurre al enfoque histórico-lógico de la literatura especializada, con el objeto de revisar el aporte recíproco de los sistemas socio-ecológicos y la resiliencia. Enseguida, se utiliza el método de análisis-síntesis, conveniente al identificar la importancia y el alcance de los elementos de un marco referencial (análisis). Luego, se unifican criterios semejantes para conectar relaciones y formar un todo (síntesis), como aporte al conocimiento científico.

El artículo se compone de varios apartados. Primero, se señala la trascendencia de los sistemas socio-ecológicos y su vínculo con la resiliencia. Asimismo, se detalla una progresión de las definiciones de resiliencia, hasta converger con una caracterización de sus dimensiones operativas, las cuales, en conjunto, denotan la riqueza de su fundamentación teórica. Luego, se describe el proceso metodológico, tanto de la recuperación de las investigaciones especializadas en la temática como de la deconstrucción del marco metodológico. A continuación, se precisan los resultados del análisis de la literatura especializada en resiliencia y turismo. Al respecto, se destacan, acompañados de un conjunto de ejemplos, los elementos clave de los sistemas socio-ecológicos y la utilidad de usar factores de resiliencia. También se muestran los aportes y potencialidades de los marcos teóricos, con enfoque en la localidad. Posteriormente, se detalla la modelización de la propuesta metodológica para estimar la resiliencia. Finalmente, se reflexiona sobre los aspectos que fortalecen el estudio de la resiliencia, la importancia de una herramienta metodológica y el surgimiento de nuevas exigencias en este campo de estudio. 


\section{Revisión de la literatura sobre resiliencia socio-ecológica}

\subsection{Sistemas socio-ecológicos (SSE)}

El término sistema socio-ecológico enfatiza el concepto integrado humano-naturaleza, así obtiene análisis profundos de la realidad. Se destacan como escenarios complejos con elementos dinámicos y de múltiples relaciones, donde uno de sus principales atributos es la resiliencia. En la praxis, un sistema socio-ecológico es una comunidad de personas con dependencia de los recursos naturales, que comprende sus relaciones socio-ecológicas desde una perspectiva sistémica, institucional y de poder. Aquí, la dinámica social en su propio territorio es la que dibuja procesos de resiliencia para manejar la incertidumbre y responder de forma creativa a la perturbación, con el propósito de delinear su desarrollo (Chontasi Morales y Ortega Vásconez, 2020).

\subsection{Resiliencia ecológica-ingeniería}

Interpretar las características de la resiliencia desde la perspectiva sistémica es importante, por tanto, es preciso comprender el campo de acción de la resiliencia desde el punto de vista de la ingeniería y la ecología, sobre todo, si se persigue avanzar en el debate científico y el desarrollo del conocimiento (Tabla 1).

La resiliencia ecológica "es una medida de la persistencia de los sistemas y de su capacidad para absorber los cambios y las perturbaciones, y mantener las mismas relaciones entre las poblaciones o las variables de estado" (Holling, 1973, p. 14). Este tipo de resiliencia es parte de la ecología moderna porque reconoce el comportamiento de un estado dinámico, cuya medida es la adaptación, entendida como la capacidad de la sociedad para controlar el cambio para influir en la recuperación; y la transformación, que se refiere a la capacidad de crear un sistema nuevo cuando las condiciones ecológicas, económicas o sociales (incluidas las políticas) hacen insostenible el sistema existente (Walker et al., 2004).

La resiliencia ecológica reconoce que las sociedades no son sistemas lineales o equilibrados, sino que son sistemas heterogéneos, con múltiples interrelaciones, propensos a recibir sorpresas y aceptar los cambios como una oportunidad. También es un marco teórico útil para identificar y evaluar comportamientos de los actores que aumentan o atentan a su capacidad de resiliencia y, por ende, a su sostenibilidad.

La resiliencia de ingeniería es "la capacidad de un sistema para retornar a un estado de equilibrio después de una perturbación temporal" (Holling, 1973, p. 14). Su base fundamental es conocer el grado de resistencia y la velocidad de un sistema para regresar a su estado inicial, es decir, qué tan rápido se movió y cuánto demoró en regresar a su estabilidad (Gunderson, 2000). Se alinea al pensamiento que reconoce al equilibrio como único estado y debe evitarse la existencia de algún otro. La perspectiva operativa percibe, en esta resiliencia, un enfoque optimista ante una crisis, pues permite a los actores dar respuestas a prueba de fallas, con base en preceptos de eficiencia predecible, constancia y control (Davoudi et al., 2012; Folke, 2006; Holling, 1996). La resiliencia de ingeniería se orienta hacia organizaciones o comunidades afectadas por desastres, crisis económicas, sociales o políticas que, a través del planteamiento de objetivos de óptimo desarrollo, buscan la recuperación inmediata de sus fuerzas económicas y estructurales, con la visión de satisfacer las necesidades del presente y del futuro, sin ningún tipo de restricción a cambio.

Tabla 1. Características de la resiliencia Table 1. Characteristics of resilience

\begin{tabular}{ll}
\hline Perspectiva de ingeniería & Perspectiva ecológica \\
\hline Tiene enfoque lineal, busca el equilibrio. & Enfoque no lineal, acepta el cambio. \\
Eficiencia en control de recursos. & Los recursos se autorregulan. \\
El cambio es reversible. & El cambio es irreversible. \\
Aplicado a infraestructura. & Aplica a poblaciones orgánicas. \\
El tiempo es clave para la recuperación. & No depende del tiempo, sino de la adaptación. \\
Busca objetivos óptimos de desarrollo. & Aporta a objetivos sostenibles. \\
Limitada diversidad. & La diversidad es clave. \\
Busca regresar al punto de partida. & Se adapta o se transforma. \\
\hline
\end{tabular}




\subsection{Marcos teóricos-metodológicos de resiliencia}

La resiliencia socio-ecológica es mucho más que un concepto. Es una herramienta analítica para comprender la realidad objetiva (Ruiz-Ballesteros, 2013). La comunidad científica ha vinculado sus esfuerzos para homologar criterios e indicadores que evalúen la resiliencia, donde se destaca el ciclo adaptativo como un diagrama tridimensional del cambio y la transformación de un sistema socio-ecológico en el tiempo y el espacio, a través de cuatro estados: conservación, deliberación, reorganización y explotación (Allen et al., 2014; Gunderson y Holling, 2002). La organización internacional Resilience Alliance (RA) y el Stockholm Resillience Centre (SRC), especializada en la investigación de la resiliencia y las ciencias de la sostenibilidad, han modelado marcos descriptivos del aumento o disminución de la resiliencia. RA propone definir el sistema, identificar dinámicas, interacciones, tipos de gobernanza y criterios de evaluación (Resilience Alliance, 2010). SRC determinó siete principios que fortalecen la visión socio-ecológica diversidad y redundancia, conectividad, variables de cambio, pensamiento adaptativo complejo, aprendizaje, participación y gobernanza (Stockholm Resilience Centre, 2020). Marcos analíticos que en los últimos años han venido siendo adaptados a la realidad del turismo con visión local (Chontasi Morales y Ortega Vásconez, 2020; Espeso-Molinero y Pastor-Alfonso, 2020; Kline, 2017; Sisneros-Kidd et al., 2019; Tsao y Ni, 2016).

\subsection{Resiliencia y turismo comunitario}

El dominio del conocimiento del turismo sostenible ha hecho muy poco uso de los avances de la investigación en los campos de los sistemas socio-ecológicos. El turismo, sobre todo el turismo comunitario, es un excelente ejemplo de SSE porque es dinámico, reconfigura las relaciones socio-ecológicas (Farrell y Twining-Ward, 2004). Es decir, la relación de la gente con los sistemas productivos, las especies y el lugar. Estas configuraciones surgen de las relaciones de poder por el control, el uso, la representación y acceso a los recursos naturales (Del Cairo et al., 2018).

Los territorios pequeños que dependen de los recursos naturales invierten más en su sostenibilidad, generan nuevos estilos de vida y de consumo que impulsan una economía social que vela por la gente y no únicamente por las ganancias, y es más probable que la autoorganización funcione mucho mejor que las políticas gubernamentales (Azkarraga Etxagibel et al., 2012). Además, a través del turismo, las comunidades locales adquieren un doble mandato: proteger los valores socioculturales y naturales, y garantizar la gestión del turismo (González-Romero et al., 2018).

El turismo comunitario es una estrategia para desarrollar una gestión resiliente que ayude a superar perturbaciones como desastres naturales, conflictos sociales, crisis económica, así como la posibilidad de restauración ambiental (Escalera-Reyes y Ruiz-Ballesteros, 2011). Desde su concepción teórica el turismo comunitario ha evolucionado (Murphy, 1985), al punto de ser considerado una estrategia de sostenibilidad, donde la comunidad gestiona sus recursos, participa y se empodera (Giampiccoli y Mtapuri, 2012; Scheyvens, 2015; Tosun, 2000) y los turistas aumentan su conciencia socio-ambiental y aprenden sobre la comunidad y las forma de vida locales (Suansri, 2003). Éstos son aspectos claves para una mejor comprensión del aumento o bloqueo de la resiliencia. Siendo este último, también, un tema de debate en los nuevos paradigmas de la investigación turística (Becken, 2013).

\section{Metodología}

Desarrollar la propuesta metodológica exigió recurrir a una serie de pasos obligados. Se partió de una investigación bibliográfica y documental de manera independiente, cuya finalidad fue relacionar la fundamentación teórica de resiliencia con la actualización científica y la aplicación en el área del turismo. La búsqueda de la información fue exhaustiva y eficaz. En la selección del material se dio preferencia a los artículos en inglés, con alto índice de citas y de revistas científicas alojadas en bases de datos multidisciplinares (Scopus y Web of Science). Asimismo, se privilegiaron libros o capítulos de libros de carácter científico y con prestigio editorial, cuya difusión fuera equivalente a una revista o artículo científico de calidad. A fin de garantizar el desarrollo ordenado de la investigación, se recurrió al carácter histórico-lógico. El propósito fue identificar la trayectoria conceptual y las principales características de la resiliencia en sistemas socio-ecológicos, para contribuir al objetivo general de la investigación. 
Un marco analítico o framework es un conjunto de criterios con la función de ayudar a simplificar y dar lógica a la realidad objetiva. Precisamente, se traduce como "una guía para identificar y analizar las múltiples posibilidades que dan forma a la vulnerabilidad, la resiliencia, el cambio en un sistema socio-ecológico del cual el turismo es parte" (Calgaro et al., 2014, p. 347). El marco analítico se constituye en un agente o enlace de conocimiento entre los usuarios (Jacobson et al., 2003). Hace posible identificar un conjunto común de variables potencialmente relevantes para convertir lo abstracto de la resiliencia en propiedades concretas (Masten, 2002), y para generar estudios longitudinales de carácter multidisciplinar que mejoran la calidad integral de la resiliencia y no caer en reduccionismos.

Desde esta perspectiva, se deconstruyó un marco analítico dinámico de valor cualitativo, con base en el uso de la lógica y la creatividad, procurando facilitar el aprendizaje y la comprensión recíproca de la resiliencia socio-ecológica en contextos locales. Por ello, fue de gran beneficio el método de análisis-síntesis, porque ambos aspectos actúan íntimamente intrincados para distinguir, sin separar, los elementos del sistema socio-ecológico y, posteriormente, combinar sus relaciones y semejanzas en un todo hermenéutico. En continuidad con este postulado, se identificaron y describieron los elementos dinámicos de un sistema socio-ecológico que influyen en la resiliencia. Luego, se reconocieron en la literatura, respecto a resiliencia y turismo, argumentos o propuestas de marcos teórico-metodológicos, cuya formulación responde a las dinámicas locales en el contexto de cambio. Entre ellos, destacan los aportes al objeto de estudio y el uso de factores de resiliencia como estrategia innovadora. Finalmente, se diseñó una propuesta de marco teórico-metodológico de resiliencia, eficaz con la voluntad de ayudar a la investigación, al incremento de la teoría y a la experimentación de la resiliencia en comunidad y en turismo comunitario.

\section{Resultados y discusión}

\subsection{Elementos dinámicos de un sistema socio-ecológico}

Los sistemas socio-ecológicos tienen el reto de evaluar el comportamiento de sus interrelaciones, para clarificar su estado y definir su capacidad de confrontar fuerzas internas y externas que atentan contra su funcionamiento. Las interacciones al interior del sistema definen qué elementos ayudan a observar la magnitud, el aprendizaje, las decisiones y la redundancia alrededor del cambio y la incertidumbre. En este contexto, las relaciones socio-ecológicas producen dinámicas y actitudes proactivas para sostener, a lo largo del tiempo, la resiliencia del sistema (Folke, 2006). Así, las amplias aportaciones teóricas sobre sistemas dinámicos relacionan a cuatro elementos: perturbación, diversidad, conocimiento, autoorganización (Carpenter et al., 2001; Folke, 2006).

La perturbación, evento natural o antrópico, condiciona y expone el comportamiento del sistema a conflictos, riesgos o vulnerabilidades. Desde la visión socio-ecológica, es la fuerza esencial para el cambio, o bien, la oportunidad de beneficiarse de él, pues no se ve únicamente como un proceso tradicionalmente negativo (Carpenter et al., 2001). Por tratarse de una acción natural y antrópica, la perturbación siempre ha existido, sin embargo, en los últimos años se incrementó por las diferentes prácticas expansivas de la humanidad, expresándose, principalmente, en el calentamiento global (Becken, 2013).

Un sistema dinámico se fundamenta en la diversidad de interrelaciones entre cada uno de sus componentes. En este sentido, la diversidad no se confunde con simplicidad, se trata de una noción prolija de conexión centralizada de respuestas para lidiar con las fuerzas perturbadoras. Desarrolla capacidades de almacenamiento y mitigación de las cuales disponer cuando el sistema sufra imprevistos o efectos acumulativos por una mala gestión (Davidson, 2010). La diversidad desarrolla respuestas adaptativas y mejora las posibilidades de encarar el cambio. Este elemento del SSE se genera cuando el humano gestiona ventajas efectivas, producto de factores como expansión de los límites ecológicos y culturales, descentralización, pluralismo, tolerancia política, niveles de complejidad, dinámicas y actitudes proactivas (Turner et al., 2003). Por consiguiente, la variedad de recursos y capitales conduce a la autosuficiencia, al conocimiento y a la innovación del sistema, y ayuda a generar estimaciones futuras.

El conocimiento es la comprensión y el poder explicativo de la historia natural y social del sistema. Hoy, en especial, es imprescindible el conocimiento, porque ayuda a comprender los sucesos y a saber cómo responder a todas aquellas manifestaciones amenazantes o riesgosas para la humanidad y su interrelación con la naturaleza. De este modo, las "organizaciones ya no dependen de sus recursos tangibles, sino más bien de la 
gestión del capital de conocimiento" (Paraskevas et al., 2013, p. 131), el cual se compone y complementa por el conocimiento tradicional y científico. Los procesos de gobernanza incorporan el conocimiento ecológico a su capital cultural y permiten mostrar anticipación y coherencia a la hora de encarar la incertidumbre y la renovación, pero, sobre todo, impiden al cambio afectar la función e identidad del sistema (Berkes, 2007; Carpenter et al., 2001; Berkes et al., 2002).

La autoorganización es clave en un sistema humano-ambiental, porque revela la complejidad de las instituciones, quienes antes de querer proteger, prefieren cambiar y alcanzar algo nuevo o mejor. A través del policentrismo, las instituciones se organizan de mejor manera en lo relativo a los procesos de adaptación y transformación, recurren a la memoria socio-ecológica del sistema y, con esto, desarrollan la capacidad adaptativa (Folke et al., 2005). De esta manera, la autoorganización dota de importancia a la colaboración interna (desde adentro) para manejar la autonomía y su identidad original. En el interior de una localidad turística, la autoorganización es una "fuerza de impulso socio-económica que genera impactos en la humanidad y la naturaleza" (Holling et al., 1995, p. 44), por lo tanto, la toma de decisiones debe ser de forma independiente y ajena a presiones políticas externas, solo así lleva al sistema a un estado próspero o decadente.

\subsection{Factores que aumentan la resiliencia}

Ningún mecanismo garantiza el mantenimiento de la resiliencia, porque ésta puede cambiar con el tiempo y su capacidad puede no ser directamente observable, con lo cual se pierde el reconocimiento de sus aspectos. Razón por la cual aparecen factores de resiliencia, con el propósito de reflexionar de manera prospectiva a las virtudes de la resiliencia.

No son indicadores, sino estimadores cualitativos coherentes de las características abstractas de la resiliencia, de tal forma que ayudan a los usuarios, si la desconocen, a entender la capacidad de la resiliencia o a recibir la misma información de una evaluación (Carpenter et al., 2005). En la Tabla 2 se distingue ejemplos de factores de resiliencia que actúan como elementos intermediarios entre los elementos del sistema socio-ecológico y las variables interpretativas de resiliencia.

Tabla 2. Factores de resiliencia

Table 2. Factors of resilience

\begin{tabular}{ll}
\hline Factores identificados & Autor \\
\hline $\begin{array}{l}\text { Presupuestos del gobierno local, conocimiento ambiental, bienestar comunitario, apoyo } \\
\text { social. }\end{array}$ & (Lew et al., 2016) \\
$\begin{array}{ll}\text { Aprender a vivir con el cambio y la incertidumbre, fomentar la diversidad, combinar dife- } \\
\text { rentes tipos de conocimientos y crear oportunidades para la reorganización. }\end{array}$ & $\begin{array}{l}\text { (Berkes, 2007; Berkes } \\
\text { et al., 2002) }\end{array}$ \\
\hline
\end{tabular}

\subsection{Análisis de marcos teórico-metodológicos (aportes-principios-potencialidades)}

Los académicos constantemente necesitan traducir el conocimiento y el impacto de los resultados de las investigaciones, en ese afán, el marco teórico-metodológico resulta un conductor apropiado para representar la teoría y la correspondiente identificación de dimensiones clave, de acuerdo con el uso apropiado y responsable de datos y evidencias (Coburn y Turner, 2011; Kagan et al., 2008). El interés de muchos autores se evidencia cada vez más, y han traducido sus investigaciones con marcos metódicos capaces de explicar lo que aún se desconoce y seleccionar los aprendizajes importantes y necesarios (Jacobson et al., 2003). En consecuencia, un marco teórico-metodológico es una estructura sistémica-lógica con el propósito de comprender el problema de estudio y obtener conocimiento nuevo. "Ayuda a los investigadores nuevos o experimentados a organizar, conceptualizar y llevar a cabo su investigación, ya sea cualitativa, cuantitativa o mixta" (Rocco y Plakhotnik, 2009, p. 120).

El turismo centró su atención en explicar, a través de marcos metodológicos, la manera de operar la sostenibilidad. No obstante, surgió el pensamiento de evaluar al turismo desde la perspectiva de resiliencia socio-ecológica, convirtiéndose en una experiencia nueva y emergente (Becken, 2013; Biggs, 2011; Lew, 2013). Con la revisión de la literatura se encontraron doce estudios, cuyas propuestas de marcos teórico-me- 
todológicos (ver Tabla 3) demostraron el uso del proceso de evaluación de la resiliencia con distintos fines: investigar los impactos del turismo, diferenciar indicadores medibles entre sostenibilidad y resiliencia, destacar las dimensiones y dominios de la resiliencia en el turismo, observar principios para desarrollar resiliencia, ponderar las capacidades colaborativas como estrategia de resiliencia y determinar los factores que aumentan la resiliencia. El esquema común fue presentar una dimensión superior donde se alojan elementos fijos, los cuales conducen a representar dinámicas, indicadores, medidas o acciones, en vista del análisis y la representación de una situación real y en constante actualización.

La Tabla 3 detalla aquellos modelos que evalúan el impacto del turismo a partir de las características de un sistema socio-ecológico y las propiedades de la resiliencia. La perturbación encarna de forma recurrente el motivo de cambio y evolución. En tanto la gobernanza y la institucionalidad son factores fundamentales a la hora de evaluar, pues su presencia permite a las estructuras ser formales y flexibles.

Tabla 3. Marcos teórico-metodológicos de resiliencia

Table 3. Theoretical-methodological frameworks of resilience

\begin{tabular}{llll}
\hline $\mathbf{N}^{\mathbf{0}}$ & Nombre de la propuesta analítica & Enfoque & Autor \\
\hline 2 & Cuatro dimensiones de la resiliencia. & $\begin{array}{l}\text { Turismo comunitario } \\
\text { Cambio climático }\end{array}$ & (Holladay y Powell, 2013) \\
& & Comunidad & (Emery y Flora, 2006) \\
3 & Marco de capitales comunitarios. & Comunidad & (Lew et al., 2016) \\
4 & Indicadores de sostenibilidad y resiliencia. & Destinos sostenibles & (Calgaro et al., 2014) \\
5 & Elementos del destino turístico sostenible. & Turismo comunitario & (Berkes et al., 2002) \\
\hline
\end{tabular}

\subsection{Propuesta de marco dinámico de resiliencia para el análisis a escala local}

La propuesta de marco dinámico de resiliencia para territorios locales (Tabla 4), se basa en dos dominios conceptuales: sistemas socio-ecológicos y resiliencia con relación al turismo. A su vez, se divide en dos niveles, con un bloque intermedio que recoge, a diferente escala, los atributos de la resiliencia.

\subsubsection{Primer nivel: anclajes estructurales-factores de resiliencia}

La perturbación, la diversidad, el conocimiento y la autoorganización son elementos que tienen la particularidad de ser permanentes dentro de cualquier estado del sistema y estar sometidos a desafíos de resistencia y adaptación. Para la presente propuesta, actúan como anclajes estructurales, tienen la función de conectar los factores de resiliencia con las dinámicas que la aumentan o la disminuyen. Es decir, son puntos estratégicos donde se desarrolla el proceso de adaptación y transformación del estado del sistema.

Recurrir al uso de factores de resiliencia, es una forma innovadora para extraer las características abstractas o aquellas que pasan desapercibidas. Estos factores, también llamados proxies, permiten aumentar la resiliencia (Carpenter et al., 2005). La funcionalidad de éstos dentro de la propuesta teórico-metodológica está en su característica de ser intermediarios y recibir las necesidades de la resiliencia, lo cual evita centrar la atención únicamente en indicadores resilientes y considerar también su forma de actuar.

Los cuatro factores de resiliencia propuestos por Berkes et al. (2002, pp. 354-355) son idóneos para el enfoque local y del turismo comunitario (Escalera-Reyes y Ruiz-Ballesteros, 2011; Ruiz-Ballesteros, 2011; Ruiz-Ballesteros y del Campo Tejedor, 2020). Conforme a este planteamiento: (1) aprender a vivir con el cambio y la incertidumbre supone asumir y enfrentar la perturbación y lo inesperado. Con el desarrollo de actitudes resilientes y prácticas adaptativas, el cambio se visualiza como una oportunidad. (2) Fomentar la diversidad implica nutrir y mejorar la memoria social y ecológica, para desarrollar opciones de respuestas flexibles y oportunas. (3) Combinar diferentes tipos de conocimiento en beneficio el aprendizaje, en esa dinámica destaca el intercambio colaborativo entre locales y científicos, con el objeto de desarrollar un conocimiento complementario de acuerdo con las necesidades del territorio. (4) Crear oportunidades para la reorganización, en las cuales se distingan las dinámicas endógenas como respuesta a las perturbaciones. 
Tabla 4. Propuesta de marco dinámico de resiliencia socio-ecológica adaptado al turismo comunitario (MDR). Table 4. Proposal for a dynamic socio-ecological resilience framework adapted to community-based tourism (MDR).

\begin{tabular}{cc}
\hline \multicolumn{2}{c}{ Elementos fijos } \\
\hline $\begin{array}{c}\text { Anclaje } \\
\text { estructural }\end{array}$ & $\begin{array}{c}\text { Sustituto de } \\
\text { resiliencia }\end{array}$ \\
\hline
\end{tabular}

$\begin{array}{ll}\text { Perturbación } & \begin{array}{l}\text { Aprender a vivir } \\ \text { con el cambio y } \\ \text { la incertidumbre. }\end{array}\end{array}$
perturbaciones, del cambio).

\section{Derivaciones resilientes}

\section{Derivaciones y dinámicas de resiliencia}

Dinámicas resilientes

Preguntas guía estrés (fuerza

Variables de cambio rápido: inundaciones, terrorismo, pandemias, tsunamis, crisis económicas, terremotos.

Variables de cambio lento: cambio climático, escasez de agua, migración económica, consumo de combustibles fósiles, uso inapropiado de la tierra, problemas públicos.

Capital natural: reducir la presión a recursos naturales, praxis consciente con el entorno, gestión responsab le de bienes naturales (cuerpos de agua, topografía, geología, flora, fauna, paisajes).

Capital cultural: revitalización del patrimonio cultural (memoria oral, sitios históricos, gastronomía, producción simbólica).

Gestión de capitales

Diversidad Fomentar la diversidad.

Capital social: bonding, bridging, linking. territoriales (neutraliza la dependencia).

Capital financiero: microfinanciación local, educación financiera, capacidad de ahorro.

Capital económico: nuevas actividades alternativas, empleo, proyectos locales (turismo, microempresas, restaurantes, huertos comunitarios)

Capital político: normas y políticas de configuración socio-ecológica.

Capital de infraestructura: carreteras, líneas de alcantarillado, reservorios, edificios, arquitectura local.

Validación de conocimiento tradicional.

Usos innovadores del conocimiento tradicional.

Transferencia Participación integral en la

Combinar

Conocimiento

\section{diferentes tipos de} conocimiento. de conocimiento (firmeza para encarar el cambio). transferencia del conocimiento.

Creación de nuevos conocimientos de interés público.

Procesos de retroalimentación.
¿Cuáles son las principales preocupaciones del presente y futuro de la comunidad?

¿Qué factores atentan la unión de la comunidad?

¿Los problemas de la comunidad son idénticos a los de localidades aledañas?

¿A qué amenazas naturales está expuesta la comunidad?

¿A qué amenazas sociales y económicas está expuesta la comunidad?

¿Cómo se usan de manera productiva los recursos naturales y culturales?

¿Qué alianzas tienen con las comunidades vecinas o amigas?

¿Qué tipos de ayuda e incentivos se generan dentro de la comunidad?

¿Existe una red familiar fuerte más allá de padres e hijos?

¿Es necesaria la asistencia externa al desarrollo comunitario?

¿Cómo obtienen créditos financieros?

¿De qué forma se desarrolla el efecto multiplicador dentro de la comunidad (reinversión local)?

¿Qué actividades productivas aparecieron en los últimos años?

¿Qué otros oficios se generan alrededor de las nuevas actividades productivas o económicas?

¿Qué porcentaje representa la actividad alternativa en los ingresos económicos?

¿Qué bienes comunes posee la comunidad?

¿Cómo se gestiona la adquisición y el uso de bienes comunes?

¿Se considera una línea de tiempo de las perturbaciones y se compara con las preocupaciones actuales?

¿Cuánto tiempo podrían operar después de un choque social o ambiental?

¿El apoyo técnico y científico avala sus actividades productivas, económicas y cotidianas?

¿Qué tipos de efectos generan las actividades productivas y cotidianas en su entorno?

¿Qué decisiones se tomaron o evitaron y cuáles fueron sus consecuencias?

¿Se transfiere la memoria socio-ecológica a las nuevas generaciones de la comunidad?

¿Hay programas de capacitación para los habitantes? 


\begin{tabular}{|c|c|c|c|c|}
\hline \multirow{12}{*}{ Autoorganización } & \multirow{12}{*}{$\begin{array}{l}\text { Crear } \\
\text { oportunidades } \\
\text { para la } \\
\text { autoorganización. }\end{array}$} & \multirow{12}{*}{$\begin{array}{l}\text { Modelos } \\
\text { endógenos de } \\
\text { organización } \\
\text { y gestión } \\
\text { de recursos } \\
\text { (innovar antes } \\
\text { que conservar). }\end{array}$} & Gobernanza policéntrica. & $\begin{array}{l}\text { ¿Cómo se encuentra organizada social y } \\
\text { administrativamente la comunidad? }\end{array}$ \\
\hline & & & $\begin{array}{l}\text { Autonomía para elaborar y hacer } \\
\text { cumplir sus reglas. }\end{array}$ & $\begin{array}{l}\text { ¿Cómo funciona el sistema de rendición de } \\
\text { cuentas? }\end{array}$ \\
\hline & & & \multirow{3}{*}{$\begin{array}{l}\text { Creación de organizaciones de } \\
\text { segundo grado (junta de agua, de } \\
\text { seguridad, turismo comunitario, } \\
\text { grupos culturales, asociaciones). }\end{array}$} & $\begin{array}{l}\text { ¿La gestión de bienes comunitarios es una } \\
\text { prioridad y estrategia de la organización? }\end{array}$ \\
\hline & & & & $\begin{array}{l}\text { ¿Qué temas se discuten y cómo se toman } \\
\text { decisiones en asamblea? }\end{array}$ \\
\hline & & & & ¿La estructura de poder tiene visión pluralista? \\
\hline & & & \multirow{2}{*}{$\begin{array}{l}\text { Gestión de zonas de interés cultural } \\
\text { y natural (áreas protegidas, sitios } \\
\text { históricos, arqueológicos). }\end{array}$} & $\begin{array}{l}\text { ¿Las mujeres tienen un papel protagónico } \\
\text { dentro de la comunidad? }\end{array}$ \\
\hline & & & & $\begin{array}{l}\text { ¿Cómo y cuándo se distribuye el trabajo } \\
\text { colectivo en la comunidad (periodos de trabajo } \\
\text { colectivo, minga)? }\end{array}$ \\
\hline & & & $\begin{array}{l}\text { Libertad para la creatividad y la } \\
\text { innovación. }\end{array}$ & $\begin{array}{l}\text { ¿Qué tipo de políticas y normas se } \\
\text { implementan en la comunidad? }\end{array}$ \\
\hline & & & \multirow{4}{*}{$\begin{array}{l}\text { Actitudes resilientes para la } \\
\text { organización (confianza, } \\
\text { reciprocidad, comunicación). }\end{array}$} & $\begin{array}{l}\text { ¿Qué papel juegan las organizaciones de } \\
\text { segundo grado? }\end{array}$ \\
\hline & & & & $\begin{array}{l}\text { ¿Qué privilegios concedieron las autoridades } \\
\text { seccionales a la comunidad? }\end{array}$ \\
\hline & & & & $\begin{array}{l}\text { ¿Los diferentes usos del territorio se hacen con } \\
\text { la aprobación de la comunidad? }\end{array}$ \\
\hline & & & & $\begin{array}{l}\text { ¿Qué lógicas no comunitarias se experimentan } \\
\text { en la comunidad? }\end{array}$ \\
\hline
\end{tabular}

Fuente: Elaborado a partir de Berkes, 2007; Berkes et al., 2002; Escalera-Reyes y Ruiz-Ballesteros, 2011; Ruiz-Ballesteros, 2011.

\subsubsection{Segundo nivel: derivaciones y dinámicas resilientes}

a) Interpretar la perturbación-gestión de la diversidad por capitales

La perturbación o también presiones internas o externas derivan de comprender si su impacto es de escala local, regional o internacional, y de reconocer dónde ocurrió el cambio y a qué velocidad. La exposición al riesgo debe medirse en la siguiente escala: estrés (variable de cambio lento), perturbación (confluencia de variables de cambio rápido y lento) y choque (variable de cambio rápido) (Calgaro et al., 2014). La diversidad de un territorio se refleja en la gestión de sus capitales para ser usados o invertidos. Por tanto, aumentar la diversidad requiere pensar en la gestión integral de capitales territoriales: social (bonding, bridging, linking), humano (nuevas habilidades, conocimiento, actitud), natural (paisajes, leks, fuentes de agua), financiero (cajas comunitarias, micro financiación), cultural (gastronomías, artesanías, sitios patrimoniales), infraestructural (carreteras, reservorios, edificios, áreas comunales) y político (poder ciudadano, normas o políticas de uso y comportamiento social) (Emery y Flora, 2006; Kline, 2017).

b) Validar el conocimiento-gobernanza endógena

El fomento del aprendizaje requiere iniciativas de transferencia mediante las cuales se validen los conocimientos tradicionales, permitiéndoles ser novedosos e importantes para el habitante. De la misma forma, el conocimiento científico debe ser de interés público y alentar a la gestión de incentivos, por consiguiente, aseguran el cambio de comportamiento. Tal transferencia recíproca provocará procesos de retroalimentación y el uso adecuado del conocimiento en aras de resolver problemas y alcanzar niveles altos de resiliencia (Pelling, 2012). Las variables de cambio comportan nuevas demandas de recursos, por tanto, el sistema de gobernanza deberá desarrollar modelos endógenos de organización social y gestión de recursos. Igualmente, la formulación de políticas y normas debe contar con la inclusión y la participación activa de sus actores, en un empeño por brindar confianza y compromiso, y dar libertad a la imaginación y a la creatividad, como estrategias de transformación (Ooi et al., 2015; Tsao y Ni, 2016). 


\section{Conclusiones}

Estudiar a las comunidades turísticas como un sistema socio-ecológico, significa, primero, reconocer que son sistemas abiertos que no buscan vivir en equilibrio, sino convivir con el cambio; segundo, conceder el protagonismo al ser humano, quien robustece el papel de la institución e impulsa el desarrollo de su resiliencia; tercero, pensar que los sistemas sociales y ambientales no se pueden estudiar por separado y que juntos coevolucionan y definen su futuro; y cuarto, determinar que la perturbación, diversidad, conocimiento y autoorganización son elementos clave, porque en ellos recaen todas las fuerzas desestabilizadoras que modelan el comportamiento y la estructura del sistema. Además, cada uno de sus componentes se interrelaciona y genera mecanismos proactivos, controlan los cambios internos y encaran, de forma coherente, las coyunturas futuras (Carpenter et al., 2001).

Las investigaciones que vinculan a la resiliencia y al turismo han aumentado en la última década y se enfrentan a una pregunta principal, ¿ en qué medida el turismo puede contribuir a la capacidad resiliente de las comunidades, sobre todo, en una era de mucha inestabilidad y de eventos inesperados? Precisamente para comprender dicha capacidad, la resiliencia no solo es un concepto, sino que es un marco teórico-metodológico integral y riguroso que sirve como instrumento de evaluación de las prácticas sociales y turísticas que se desarrollan en un determinado territorio.

También ayuda a analizar cómo una comunidad concreta (SSE) funciona en su territorio y se determina qué comportamientos aportan o no a la resiliencia. De ahí que la contribución de esta propuesta es dar lógica y precisión a los comportamientos observables de un fenómeno o hecho en particular dentro del sistema, también, ayuda a estimar la resiliencia de forma cualitativa. Modelar esta herramienta dio paso a una creatividad capaz de deconstruir las posiciones que representan la realidad objetiva de una situación, incluso estereotipada.

Finalmente, la aplicación de esta perspectiva teórica-metodológica solo tomará validez cuando se aplique en estudios de caso específicos. La experimentación dará la posibilidad de ampliar y/o mejorar las variables que ayudan a identificar derivaciones y dinámicas resilientes. Así mismo, la construcción de más preguntas guías ayudarán a los investigadores a describir la relación de la resiliencia y el turismo, y a fortalecer el carácter cualitativo de los estudios.

\section{Agradecimientos}

Los autores agradecen a Pilar (Pitu) Espeso y María José Pastor investigadoras del Departamento de Humanidades Contemporáneas de la Universidad de Alicante por la supervisión. El artículo se ha beneficiado de los numerosos y útiles comentarios de los tres revisores.

\section{Financiamiento}

La presente investigación fue realizada con la colaboración y financiación de la Coordinación de Investigación Formativa (CIF) y proyectos semilla cif6-cs-fcs-2, Universidad Central del Ecuador.

\section{Contribuciones de los autores}

- Fernando David Chontasi Morales: conceptualización, curación de datos, análisis formal, investigación, metodología, adquisición de fondos, redacción - revisión y edición.

- Jessica Dayana Noguera Benalcázar: curación de datos, análisis formal, investigación, visualización.

- Daniela Pilar Ortega Vásconez: curación de datos, análisis formal, investigación, redacción - borrador original.

- Mery Tatiana Chicaiza Guaman: curación de datos, análisis formal, investigación.

- Luis Arturo Naula Morillo: curación de datos, investigación.

- Diana Carolina Duarte Victorero: curación de datos, investigación. 


\section{Referencias}

Allen, C. R., Angeler, D. G., Garmestani, A. S., Gunderson, L. H., \& Holling, C. S. (2014). Panarchy: Theory and application. Ecosystems, 17(4), 578-589. https://doi.org/10.1007/s10021-013-9744-2

Azkarraga Etxagibel, J., Sloan, T., Belloy, P., \& Loyola, A. (2012). Eco-localismos y resiliencia comunitaria frente a la crisis civilizatoria. Las iniciativas de transición. Polis, 33. http://journals.openedition.org/polis/8400

Becken, S. (2013). Developing a framework for assessing resilience of tourism sub-systems to cimatics factors. Annals of Tourism Research, 43, 506-528. https://doi.org/10.1016/j.annals.2013.06.002

Berkes, F. (2007). Understanding uncertainty and reducing vulnerability: Lessons from resilience thinking. Natural Hazards, 41, 283-295. https://doi.org/10.1007/s11069-006-9036-7

Berkes, F., Colding, J., \& Folke, C. (2002). Synthesis : Building Resilience and Adaptive Capacity in Social-Ecological Systems. In F. Berkes, J. Colding, \& C. Folke (eds.), Navigating Social-Ecological Systems : Building Resilience for Complexity and Change (1st ed., pp. 352-387). Cambridge University Press.https://doi.org/10.1017/CBO9780511541957.020

Biggs, D. (2011). Understanding resilience in a vulnerable industry: the case of reef tourism in Australia. Ecology and Society, 16(1), 30. https://doi.org/10.5751/ES-03948-160130

Calgaro, E., Lloyd, K., \& Dominey-Howes, D. (2014). From vulnerability to transformation: a framework for assessing the vulnerability and resilience of tourism destinations. Journal of Sustainable Tourism, 22(3), 341-360. https://doi.org/10.1080/09669582.2013.826229

Carpenter, S. R., Walker, B., Anderies, J. M., \& Abel, N. (2001). From Metaphor to Measurement: Resilience of What to What? Ecosystems, 4(8), 765-781. https://doi.org/10.1007/s10021-001-0045-9

Carpenter, S. R., Westley, F., \& Turner, M. G. (2005). Surrogates for resilience of social-ecological systems. Ecosystems, 8, 941-944. https://doi.org/10.1007/s10021-005-0170-y

Chontasi Morales, D., \& Ortega Vásconez, D. P. (2020). Comunidad con vocación turística: una visión desde los sistemas socio-ecológicos y la resiliencia. Gran Tour, Revista De Investigaciones Turísticas, 21, 185205. https://eutm.es/grantour/index.php/grantour/article/view/152

Coburn, C. E., \& Turner, E. O. (2011). Research on data use: a framework and analysis. Measurement: Interdisciplinary Research and Perspectives, 9(4), 173-206. https://doi.org/10.1080/15366367.2011.626729

Davidson, D. J. (2010). The applicability of the concept of resilience to social systems: Some sources of optimism and nagging doubts. Society and Natural Resources, 23(12), 1135-1149. https://doi. org/10.1080/08941921003652940

Davoudi, S., Shaw, K., Haider, L. J., Quinlan, A. E., Peterson, G. D., Wilkinson, C., Fünfgeld, H., McEvoy, D., Porter, L., \& Davoudi, S. (2012). Resilience: A Bridging Concept or a Dead End? Planning Theory \& Practice, 13(2), 299-333. https://doi.org/10.1080/14649357.2012.677124

Del Cairo, C. L., Gómez Zuñiga, S., Ortega, J. E., Ortiz Gallego, D., Rodríguez Maldonado, A. C., Vélez Triana, J. S., \& Vergara Gutiérrez, T. (2018). Dinámicas socioecológicas y ecoturismo comunitario: un análisis comparativo en el eje fluvial Guayabero-Guaviare: un análisis comparativo en el eje fluvial Guayabero-Guaviare. Cuadernos De Desarrollo Rural, 15(82), 1-23. https://doi.org/10.11144/Javeriana. cdr15-82.dsec

Emery, M., \& Flora, C. (2006). Spiraling-up: mapping community transformation with community capitals framework. Community Development, 37(1), 19-35. https://doi.org/10.1080/15575330609490152

Escalera-Reyes, J., \& Ruiz-Ballesteros, E. (2011). Resiliencia socioecológica: aportaciones y retos desde la antropología. Revista de Antropología Social, 20, 109-135. https://doi.org/10.5209/rev_RASO.2011. v20.36264

Espeso-Molinero, P., \& Pastor-Alfonso, M. J. (2020). Governance, community resilience, and indigenous tourism in Nahá, México. Sustainability, 12(15), 1-20. https://doi.org/10.3390/SU12155973

Farrell, B. H., \& Twining-Ward, L. (2004). Reconceptualizing tourism. Annals of Tourism Research, 31(2), 274-295. https://doi.org/10.1016/j.annals.2003.12.002

Folke, C. (2006). Resilience: The emergence of a perspective for social-ecological systems analyses. Global Environmental Change, 16(3), 253-267. https://doi.org/10.1016/j.gloenvcha.2006.04.002

Folke, C., Hahn, T., Olsson, P., \& Norberg, J. (2005). Adaptative governance of social-ecológical systems. Annual Review of Environment and Resources, 30(1), 441-473. https://doi.org/10.1146/annurev.energy.30.050504.144511 
Giampiccoli, A., \& Mtapuri, O. (2012). Community-based tourism: an exploration of the concept(s) from a political perspective. Tourism Review International, 16(1), 29-43. https://doi.org/10.3727/15442721 2X13431568321500

González-Romero, N. I., Galicia, L., Arteaga-Reyes, T. T., Thomé-Ortiz, H., \& Héritier, S. (2018). Recreational activities and conservation in natural protected areas in central Mexico: an approach from socio-ecosystems. Ecosistemas, 27(1), 116-126. https://doi.org/10.7818/ECOS.1407

Gunderson, L. H. (2000). Ecological resilience. In theory and application. Annual Review of Ecology and Systematics, 31(1), 425-439. https://doi.org/10.1146/annurev.ecolsys.31.1.425

Gunderson, L. H., \& Holling, C. S. (eds.). (2002). Panarchy: understanding transformations in human and natural systems. Island Press.

Holladay, P. J., \& Powell, R. B. (2013). Resident perceptions of social-ecological resilience and the sustainability of community-based tourism development in the Commonwealth of Dominica. Journal of Sustainable Tourism, 21(8), 1188-1211. https://doi.org/10.1080/09669582.2013.776059

Holling, C. S. (1973). Resilience and stability of ecological systems. Annual Review of Ecology and Systematics, 4(1), 1-23. https://doi.org/10.1146/annurev.es.04.110173.000245

Holling, C. S. (1996). Engineering resilience versus ecological resilience. In P. Pchulze (ed.), Engineering Within Ecological Constraints (pp. 31-44). National Academy Press.

Holling, C. S., Schindler, D. W., Walker, B. W., \& Roughgarden, J. (1995). Biodiversity in the functioning of ecosystems: an ecological synthesis. In C. Perrings, L. G. Mäler, C. Folke, C. S. Holling, \& B. Jansson (eds.), Biodiversity loss: economic and ecological issues (pp. 44-83). Cambridge University Press. http:// dx.doi.org/10.1017/cbo9781139174329.005

Jacobson, N., Butterill, D., \& Goering, P. (2003). Development of a framework for knowledge translation: understanding user context. Journal of Health Services Research \& Policy, 8(2), 94-99. https://doi. org/10.1258/135581903321466067

Kagan, A., Simmons-Mackie, N., Rowland, A., Huijbregts, M., Shumway, E., McEwen, S., Threats, T., \& Sharp, S. (2008). Counting what counts: A framework for capturing real-life outcomes of aphasia intervention. Aphasiology, 22(3), 258-280. https://doi.org/10.1080/02687030701282595

Kline, C. (2017). Applying the community capitals framework to the craft heritage trails of western North Carolina. Journal of Heritage Tourism, 12(5), 489-508. https://doi.org/10.1080/1743873X.2016.1226315

Lew, A. A. (2013). Scale, change and resilience in community tourism planning. Tourism Geographies, 16(1), 14-22. https://doi.org/10.1080/14616688.2013.864325

Lew, A. A., Ng, P. T., Ni, C. (Nickel), \& Wu, T. (Emily). (2016). Community sustainability and resilience: similarities, differences and indicators. Tourism Geographies, 18(1), 18-27. https://doi.org/10.1080/146 16688.2015 .1122664

Masten A. S. (2002). Resilience Comes of Age. In M. D. Glantz, \& J. L. Johnson (eds), Resilience and Development. Longitudinal Research in the Social and Behavioral Sciences: An Interdisciplinary Series (pp. 281-296). Springer. https://doi.org/10.1007/0-306-47167-1_15

Murphy, P. E. (1985). Tourism: A Community Approach. Methuen.

Ooi, N., Laing, J., \& Mair, J. (2015). Social capital as a heuristic device to explore sociocultural sustainability: a case study of mountain resort tourism in the community of Steamboat Springs, Colorado, USA. Journal of Sustainable Tourism, 23(3), 417-436. https://doi.org/10.1080/09669582.2014.957211

Paraskevas, A., Altinay, L., McLean, J., \& Cooper, C. (2013). Crisis knowledge in tourism: types, flows and governance. Annals of Tourism Research, 41, 130-152. https://doi.org/10.1016/j.annals.2012.12.005

Pelling, M. (2012). The Vulnerability of Cities: Natural Disasters and Social Resilience. Routledge.

Pickett, S. T. A., Cadenasso, M. L., \& Grove, J. M. (2004). Resilient cities: meaning, models, and metaphor for integrating the ecological, socio-economic, and planning realms. Landscape and Urban Planning, 69(4), 369-384. https://doi.org/10.1016/j.landurbplan.2003.10.035

Resilience Alliance. (2010). Assessing resilience in socia-ecological systems: workbook for practitioners (Version 2.). http://www.resalliance.org/3871.php

Rocco, T. S., \& Plakhotnik, M. S. (2009). Literature reviews, conceptual frameworks, and theoretical frameworks: terms, functions, and distinctions. Human Resource Development Review, 8(1), 120-130. https://doi.org/10.1177/1534484309332617 
Ruiz-Ballesteros, E. (2011). Social-ecological resilience and community-based tourism An approach from Agua Blanca, Ecuador. Tourism Management, 32(3), 655-666. https://doi.org/10.1016/j.tourman.2010.05.021

Ruiz-Ballesteros, E. (2013). Sociecosistemas y resiliencia socio-ecológica. Una aproximación compleja al medio ambiente. En E. Ruiz y J. Solana (eds.), Complejidad y Ciencias Sociales (pp. 295-331). Universidad de Andalucía.

Ruiz-Ballesteros, E., \& del Campo Tejedor, A. (2020). Community-based tourism as a factor in socio-ecological resilience. Economic diversification and community participation in Floreana (Galapagos). Sustainability, 12(11), 4724. https://doi.org/10.3390/su12114724

Scheyvens, R. (2015). Ecotourism and the empowerment of local communities. Tourism Management, 20(2), 245-249. https://doi.org/10.1016/S0261-5177(98)00069-7

Scoones, I. (1999). New ecology and the social sciences: what prospects for a fruitful engagement? Anual Review of Anthropology, 28(1), 479-507.

Sisneros-Kidd, A. M., Monz, C., Hausner, V., Schmidt, J., \& Clark, D. (2019). Nature-based tourism, resource dependence, and resilience of Arctic communities: framing complex issues in a changing environment. Journal of Sustainable Tourism, 27(8), 1259-1276. https://doi.org/10.1080/09669582.2019.1612905

Stockholm Resilience Centre. (2020). Seven principles for building resilience. https://www.stockholmresilience.org/research/research-news/2015-04-08-seven-principles-for-building-resilience.html

Suansri, P. (2003). Community based tourism handbook. Responsible Ecological Social Tour-REST.

Tosun, C. (2000). Limits to community participation in the tourism development process in developing countries. Tourism Management, 21(6), 613-633. https://doi.org/10.1016/S0261-5177(00)00009-1

Tsao, C., \& Ni, C. (2016). Vulnerability, resilience, and the adaptive cycle in a crisis-prone tourism community. Tourism Geographies, 18(1), 80-105. https://doi.org/10.1080/14616688.2015.1116600

Turner, N. J., Davidson-Hunt, I. J., \& O'Flaherty, M. (2003). Living on the edge: Ecological and cultural edges as sources of diversity for social-ecological resilience. Human Ecology, 31(3), 439-462. https://doi. org/10.1023/A:1025023906459

Walker, B., Holling, C. S., Carpenter, S. R., \& Kinzig, A. (2004). Resilience, Adaptability and Transformability in Social-ecological Systems. Ecology and Society, 9(2), 5. https://www.ecologyandsociety.org/vol9/ iss $2 / \operatorname{art} 5 /$ 